\title{
The Analysis of the Numerical Price Forecasting Success Considering the Modification of the Initial Condition Value by the Commodity Stock Exchanges
}

\author{
Marcela Lascsáková ${ }^{1, *}$ \\ 'Department of Applied Mathematics and Informatics, Faculty of Mechanical Engineering, Technical University of Košice, Košice, Slovak Republic
}

\begin{abstract}
In mathematical models, for forecasting prices on commodity exchanges different mathematical methods are used. In the given paper the numerical model based on the exponential approximation of commodity stock exchanges was derived. The price prognoses of aluminium on the London Metal Exchange were determined as numerical solution of the Cauchy initial problem for the $1^{\text {st }}$ order ordinary differential equation. To make the numerical model more accurate the idea of the modification of the initial condition value by the stock exchange was realized. The derived numerical model was observed to determine the success of the proposed modification by means of the distribution of the numbers of the forecasting terms with different error rate. Within the forecasting terms, in which the modification of the initial condition value by stock exchange occurred, the proposed strategy significantly improved the original forecasting. The largest forecasting problems within significant and rapid changes in price course were eliminated. The modification of the original model improved forecasting in each forecasting term where the initial condition drift occurred by reducing both the mean absolute percentage error of the forecasting term and the number of the prognoses with the absolute percentage error of at least $10 \%$.
\end{abstract}

Keywords: exponential approximation; numerical modelling; price forecasting; commodity exchange.

\section{Introduction}

One of the most important factors determining the prices of the non-ferrous metals is the impact of the London Metal Exchange (LME). It is the world's premier non-ferrous metal market. Although there are other commodity exchanges where metals are traded, the majority of producers and businessmen respect just official prices daily closed on the LME.

Observing trends and forecasting movements of metal prices is still a current problem. In mathematical models forecasting the prices on the commodity exchanges [1-12], the statistical methods [4-8] are often used. They need to process a large number of historical market data. The quantity of needed market data can sometimes be a disadvantage. In such cases, other mathematical methods are required.

In our prognostic models numerical methods are used. Derived numerical models for forecasting prices are based on the numerical solution of the Cauchy initial problem for the $1^{\text {st }}$ order ordinary differential equations [9-12]. The aluminium prices presented on the London Metal Exchange were worked on. We dealt with the 
monthly averages of the daily closing aluminium prices "Cash Seller\&Settlement price" in the period from December 2002 to June 2006 [13]. As can be seen in Figure 1, the course of the aluminium prices on the LME (in US \$ per tonne) within the considered period changed dramatically.

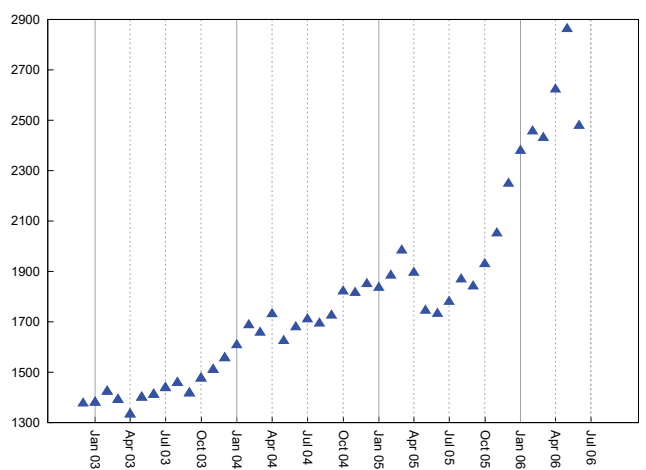

Fig. 1: Course of aluminium prices on LME in the years $2003-2006$ (in US \$ pertonne).

\section{Mathematical model} form

Let us consider the Cauchy initial problem in the $\tilde{y}=a_{0} e^{a_{1} x}$

The particular solution of the problem (1) is in the form $y=k e^{a_{1} x}$, where $k=y_{0} e^{-a_{1} x_{0}}$. The considered exponential trend was chosen according to the test criterion of the time series trend suitability. The values $\ln \left(Y_{i+1}\right)-\ln \left(Y_{i}\right)$, for $i=0$, $1, \ldots, 42$ have approximately constant course. The term $Y_{i}$ is the aluminium price (stock exchange) on LME in the month $x_{i}$.

The price prognoses were created by three steps:

$\mathbf{1}^{\text {st }}$ step: Approximation of the values - the values of the approximation term were approximated by the least squares method. The exponential function in the form $\tilde{y}=a_{0} e^{a_{1} x}$ was used. Two different variants, variant $B$ (see Figure 2 ) and variant $E$ (see Figure 3), with longer approximation terms were studied in [9]. Note that 36 forecasting terms of the original model in both variants $B$ and $E$ within period from July 2003 to June 2006 were observed. From among all forecasting terms, 11 of them belong to variant $B$ and 25 are part of variant $E$.

$2^{\text {nd }}$ step: Formulation of the Cauchy initial problem according to the acquired approximation function $\tilde{y}$, the Cauchy initial problem (1) was written in the form

$y^{\prime}=a_{1} y, \quad y\left(x_{i}\right)=Y_{i}$,

where $x_{i}=i$ is the last month of the approximation term, $Y_{i}$ is the price on LME in the month $x_{i}$.

$3^{\text {rd }}$ step: Computation of the prognoses - the formulated Cauchy initial problem (2) was solved by a numerical method [14]. The method uses the following numerical formulae

$x_{i+1}=x_{i}+h$,

$y_{i+1}=y_{i}+b h+Q e^{v x_{i}}\left(e^{v h}-1\right)$,

for $i=1,2,3, \ldots$, where $h=x_{i+1}-x_{i}$ is the constant size step. The unknown coefficients are calculated by means of these formulae

$v=\frac{f^{\prime \prime}\left(x_{i}, y_{i}\right)}{f^{\prime}\left(x_{i}, y_{i}\right)}, \quad Q=\frac{f^{\prime}\left(x_{i}, y_{i}\right)-f^{\prime \prime}\left(x_{i}, y_{i}\right)}{(1-v) v^{2} e^{v x_{i}}}$,

$b=f\left(x_{i}, y_{i}\right)-\frac{f^{\prime}\left(x_{i}, y_{i}\right)}{v}$.

If we consider the Cauchy initial problem (2), the function $f\left(x_{i}, y_{i}\right)$ has the form $f\left(x_{i}, y_{i}\right)=a_{1} y_{i}$ and then

$$
\begin{aligned}
& f^{\prime}\left(x_{i}, y_{i}\right)=a_{1} y^{\prime}\left(x_{i}\right)=a_{1}^{2} y_{i}, \\
& f^{\prime \prime}\left(x_{i}, y_{i}\right)=a_{1}^{2} y^{\prime}\left(x_{i}\right)=a_{1}^{3} y_{i} .
\end{aligned}
$$

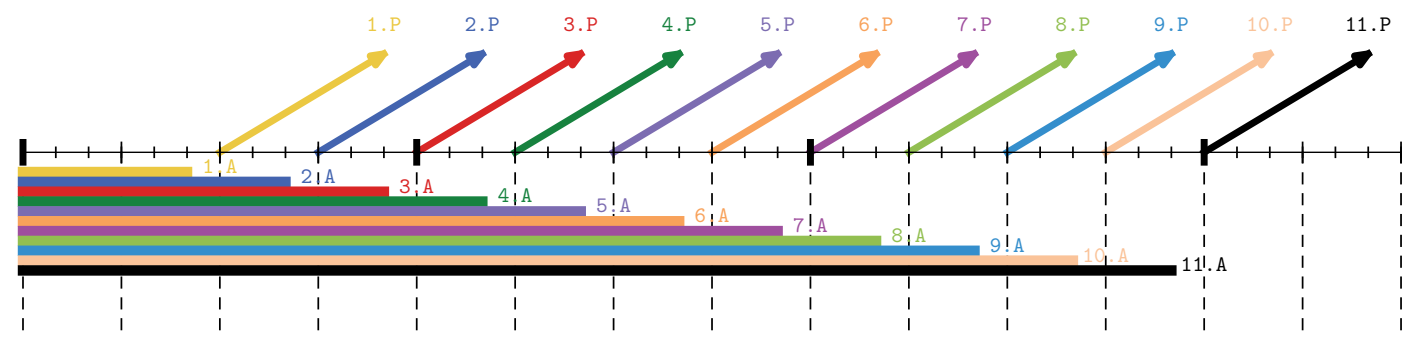

Jan-03 Apr-03 Jul-03 Oct-03 Jan-04 Apr-04 Jul-04 Oct-04 Jan-05 Apr-05 Jul-05 Oct-05 Jan-06 Apr-06 Jul-06

Fig. 2: Variant $B$ ( $A$-approximation term, $P$-forecasting term). 


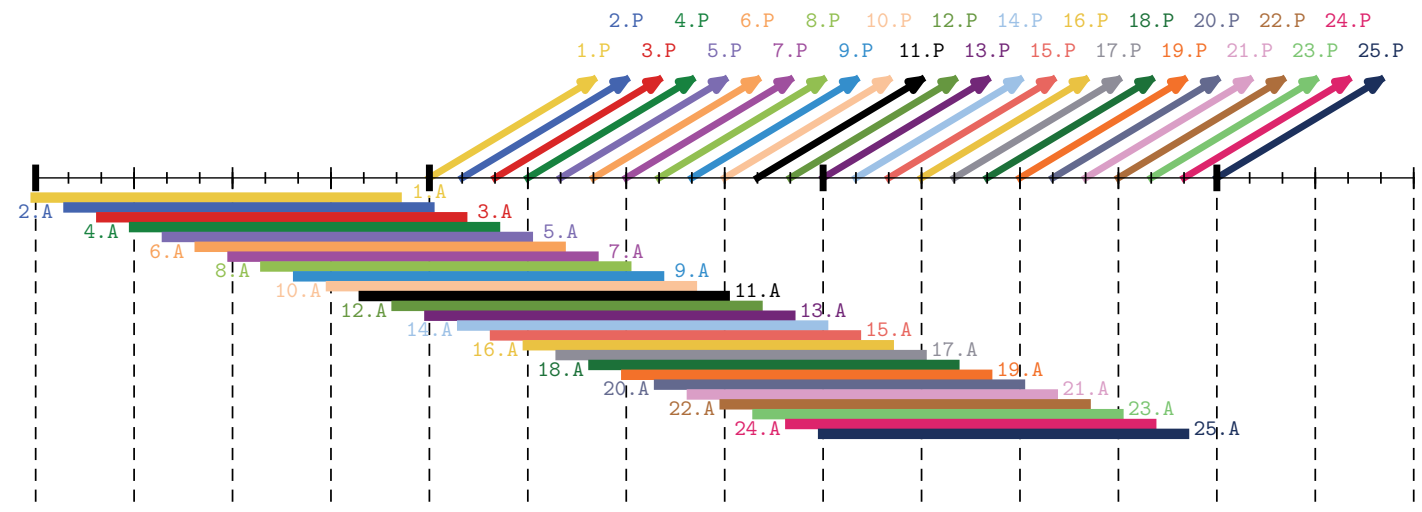

Jan-03 Apr-03 Jul-03 Oct-03 Jan-04 Apr-04 Jul-04 Oct-04 Jan-05 Apr-05 Jul-05 Oct-05 Jan-06 Apr-06 Jul-06

Fig. 3: Variant $E$ (A - approximation term, $P$-forecasting term).

The prognoses within six months that follow the end of the approximation term were calculated after modification of the initial condition value. The first month prognosis was determined by solving the Cauchy initial problem in the form (2). The interval $\left\langle x_{i}, x_{i+1}\right\rangle$ of the length $h=1$ month was divided into $n$ parts, where $n$ is the number of trading days on LME in the month $x_{i+1}$. We obtained the sequence of the division points $x_{i 0}=x_{i}, x_{i j}=x_{i}+h j / n$, for $j=1,2, \ldots, n$, where $x_{i n}=x_{i+1}$. For each point of the subdivision of the interval $\left\langle x_{i}, x_{i+1}\right\rangle$, the Cauchy initial problem in the form (2) was solved by the chosen numerical method. In this way we obtained the prognoses of the aluminium prices on single trading days $y_{i j}$ for $i=1,2, \ldots, j=1,2, \ldots, n$. By calculating the arithmetic mean of the daily prognoses we obtained the monthly prognosis of the aluminium price in the month $x_{i+1}$. So,

$$
y_{i+1}=\frac{\sum_{j=1}^{n} y_{i j}}{n} \text {. }
$$

The prognoses for the following months were calculated after modification of the initial condition value. The initial condition value in the month $x_{i+s^{\prime}}$ $s=1,2,3,4,5$ was replaced either by the calculated monthly prognosis $y_{i+s}$ or by some aluminium stock exchange (in case of higher absolute percentage error of given monthly prognosis $y_{i+s}$ ). The Cauchy initial problem $y^{\prime}=a_{1} y, y\left(x_{i+s}\right)=y_{i+s}$, or $y^{\prime}=a_{1} y$, $y\left(x_{i+s}\right)=Y_{p}$, where $Y_{p}$ is chosen aluminium stock exchange, was used to calculate daily prognoses and their arithmetic mean served to define the monthly price prognosis $y_{i+s+1}$ for the month $x_{i+s+1}$.

By comparing the calculated prognosis $y_{s}$ in the month $x_{s}$ with the real stock exchange $Y_{s^{\prime}}$ the absolute percentage error $\left|p_{s}\right|=\frac{\left|y_{s}-Y_{s}\right|}{Y_{S}} .100 \%$ was determined. The price prognosis $y_{s}$ in the month $x_{s}$ is acceptable in practice, if $\left|p_{s}\right|<10 \%$. Otherwise, it is called the critical forecasting value. To compare the accuracy of the forecasting of all forecasting terms, the mean absolute percentage error (MAPE)

$\bar{p}=\frac{\sum_{s=1}^{t}\left|p_{s}\right|}{t}$

is determined, where, in our case, $t=6$.

The modification of the initial condition value by the real aluminium stock exchange price was called the initial condition drift. Let us name the selected minimal absolute percentage error of the prognosis, causing the initial condition drift, the limiting value error. The month in which the absolute percentage error of the prognosis had at least the limiting value error was considered as the limiting month. The limiting value errors of the size $7 \%$ and $8 \%$ were chosen $[11,12]$. Let us consider three types of the initial condition drift with regard to their length [10], one-month drift, drift before the limiting month and drift to the limiting month.

\section{Results}

We started from the original model in which the prognoses in the months $x_{i+s^{\prime}}, s=2,3,4,5,6$, where 
$x_{i}$ is the last month of the approximation term, were calculated after modification of the initial condition value by the obtained monthly price prognoses [9]. Based on the prognosis accuracy analysis of the original model, forecasting terms were classified into three classes $[10,12]$ :

\section{I. trouble free forecasting terms (18 terms)}

All absolute percentage errors of the monthly prognoses within the forecasting term were less than $10 \%$.

a) The initial condition drift did not occur (14 terms) - either all absolute percentage errors of the monthly prognoses were less than $7 \%$ or the absolute percentage prognosis error in the last month of the forecasting term was from the interval $\langle 7,10)$.

b) The initial condition drift occurred (4 terms) - within observed forecasting term the absolute percentage prognosis error in some month, except the last month, was greater than or equal to $7 \%$.

II. forecasting terms with a small error (10 terms)

The mean absolute percentage error of the forecasting term was less than $10 \%$, but the absolute percentage error of some monthly prognosis was at least $10 \%$.

\section{III. forecasting terms with a big error (8 terms)}

The mean absolute percentage error of the forecasting term was at least $10 \%$.

From among 36 observed forecasting terms, a half of them were a trouble free. Approximately in 3/4 of these terms the forecasting was so accurate that the initial condition drift did not occur. The initial condition values were replaced only by calculated monthly prognoses. Thereby the original model has not changed. The second half of the original terms consisted of the forecasting terms with different errors causing the initial condition drift. This explains why the forecasting results differed from the original model. The forecasting terms with small and big errors were almost equally met.

To make the original forecasting more accurate the idea of the modification of the initial condition value by aluminium price was realized. The forecasting terms in which the initial condition drift occurred (22 terms) were taken into consideration. Within each forecasting term for the variants $B$ and $E$, the success of the forecasting within the forecasting terms with different error rate of the original model (trouble free forecasting terms, forecasting terms with a small error and forecasting terms with a big error) was studied. In both, the original model and the modified model, the changes of the number of the forecasting terms in determined groups after using the most successful initial condition drift were observed. The initial condition drift by means of which the lowest MAPE was acquired in the forecasting term was considered as the most successful. The obtained results are shown in Table 2 and Table 3.

In the variant $B$, within 11 forecasting terms the initial condition drift occurred seven times. In the

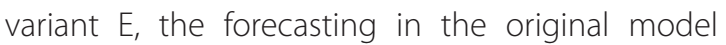
caused the initial condition drift, which was due to lesser forecasting accuracy, in 15 forecasting terms out of 25. By using determined strategy of the initial condition drift, the most problematic forecasting terms with a big error were eliminated in both variants. In these terms the original forecasting was so inaccurate that MAPE was at least $10 \%$. In all observed forecasting terms with a big error

Tab. 1: Distribution of the number of the forecasting terms in determined groups - variant $B$.

\begin{tabular}{|l|l|l|l|l|}
\hline \multirow{2}{*}{ Type of forecasting term } & \multicolumn{2}{|c|}{ Trouble free forecasting terms } & Forecasting terms with a \\
\cline { 2 - 5 } small error & $\begin{array}{c}\text { The initial condition drift } \\
\text { did not occur }\end{array}$ & $\begin{array}{c}\text { The initial condition drift } \\
\text { occurred }\end{array}$ & $\begin{array}{c}\text { Forecasting terms with a } \\
\text { big error }\end{array}$ \\
\hline The original model & 4 & 1 & 4 & 2 \\
\hline The modified model & 4 & 4 & 3 & 0 \\
\hline
\end{tabular}

Tab. 2: Distribution of the number of the forecasting terms in determined groups - variant $E$.

\begin{tabular}{|c|c|c|c|c|}
\hline \multirow[b]{2}{*}{ Type of forecasting term } & \multicolumn{2}{|c|}{ Trouble free forecasting terms } & \multirow{2}{*}{$\begin{array}{l}\text { Forecasting terms with a } \\
\text { small error }\end{array}$} & \multirow[b]{2}{*}{$\begin{array}{c}\text { Forecasting terms with a } \\
\text { big error }\end{array}$} \\
\hline & $\begin{array}{l}\text { The initial condition drift } \\
\text { did not occur }\end{array}$ & $\begin{array}{c}\text { The initial condition drift } \\
\text { occurred }\end{array}$ & & \\
\hline The original model & 10 & 3 & 6 & 6 \\
\hline The modified model & 10 & 7 & 8 & 0 \\
\hline
\end{tabular}




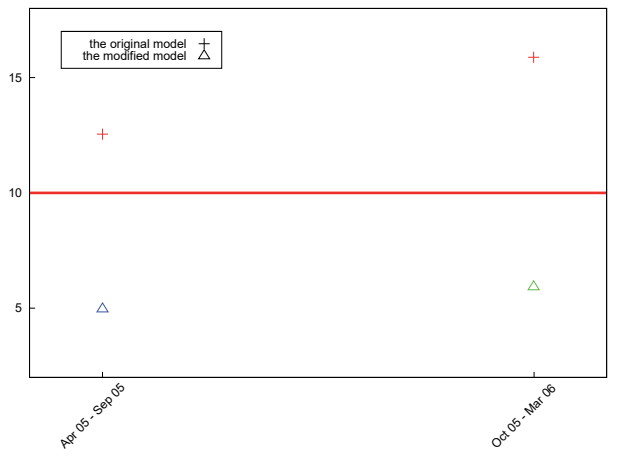

Fig. 4: The mean absolute percentage errors within the forecasting terms with a big error - variant $B$.

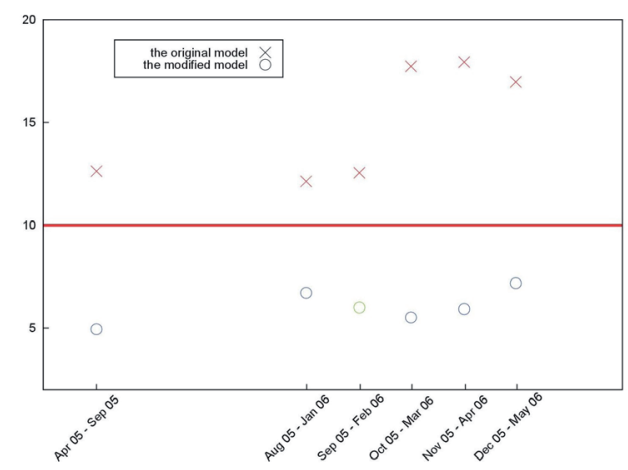

Fig. 5: The mean absolute percentage errors within the forecasting terms with a big error - variant $E$.

(8 terms), the mean absolute percentage error became less than $10 \%$ by means of the initial condition drift. In these forecasting terms of the modified model there was only one, or no critical value and this way the forecasting terms with a small error (6 terms), or even trouble free forecasting terms (2 terms) were obtained (Figure 4 and Figure 5, trouble free forecasting terms are green, forecasting terms with a small error are blue and forecasting terms with a big error are red). By means of the original forecasting, three, four or five critical values from six prognoses were acquired. That also underlines failure of the original forecasting in these terms.

After the initial condition drift, the original forecasting terms with a small error (10 terms) either became a part of trouble free forecasting terms (5 terms), which was due to elimination of all critical values, or remained in the original group of the forecasting terms with a small error (5 terms) with one critical value (Figure 6 and Figure 7). By means of the original forecasting there were three, two or one

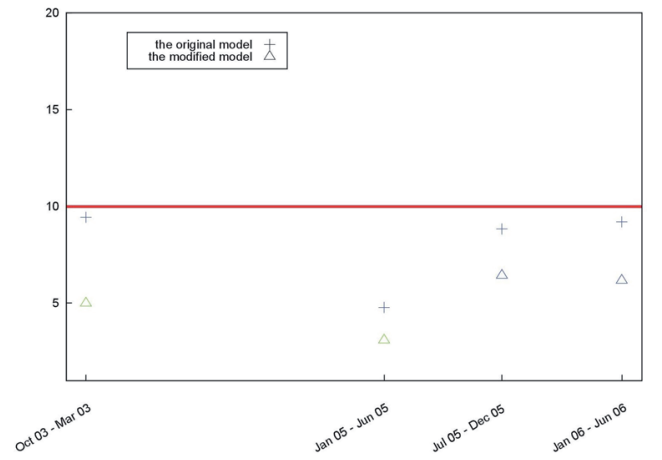

Fig. 6: The mean absolute percentage errors within the forecasting terms with a small error - variant $B$.

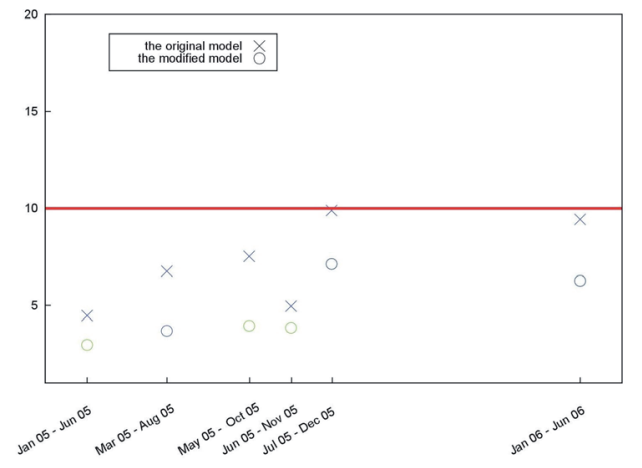

Fig. 7: The mean absolute percentage errors within the forecasting terms with a small error - variant $E$.

critical values in the forecasting terms with a small error. Anyway, the mean absolute percentage error and the number of critical values in the modified model were always reduced.

\section{Discussion}

The original model forecasted the aluminium price reliably within the stable price course, when the price did not changed rapidly. Within the rapid increase or decrease of stock exchanges, but also in the case of changes in the price course the forecasting failed. Within the original forecasting there were eight the most problematic terms with the mean absolute percentage error at least $10 \%$. In these forecasting terms with a big error there was price increase, respectively decline within the approximation terms. Therefore the approximation functions were increasing, respectively slowly increasing or decreasing, so the prognoses could not approximate steeply decreasing, respectively increasing stock exchanges. Thus, the errors of prognoses became higher, and the initial condition 


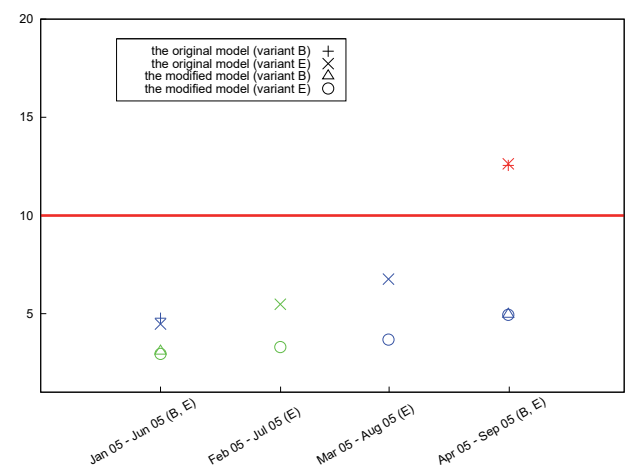

Fig. 8: The mean absolute percentage errors within price decline after price increase.

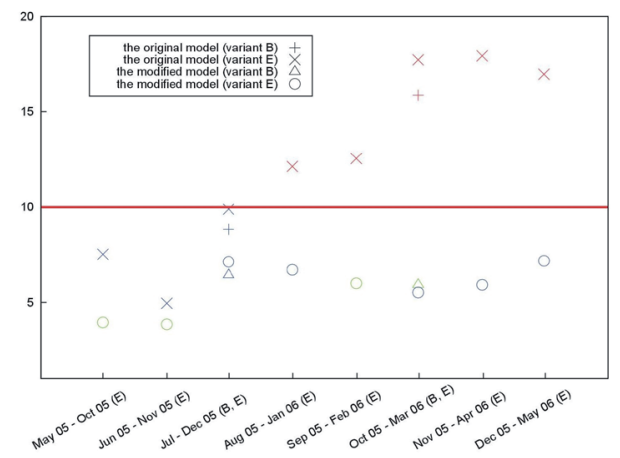

Fig. 9: The mean absolute percentage errors within price increase after price decline.

drift occurred. By using the most successful initial condition drift (the longest drift to the limiting month by means of the limiting value error $7 \%$ ), we put prognoses the nearest to the price evolution of the stock exchanges. Thanks to that, either all of the critical values were eliminated ( 2 terms), or only one critical value remained (6 terms), (Figures 8 and 9). This prognosis was applied either to month when the steep price decline after its increase occured or to the month with the steep increase of stock exchange after price decline.

Within the forecasting terms with a small error, the price increase in the forecasting term was more rapid than the price increase in approximation terms. Within price increase the prognosis error increased with time. Forecasting success was achieved by larger number of the initial condition drift due to the limiting value error $7 \%$. Thereby the prognoses by mean of the longest initial condition drift to the limiting month could better approach increasing stock exchanges, and the forecasting became more accurate. Thus, trouble free forecasting terms

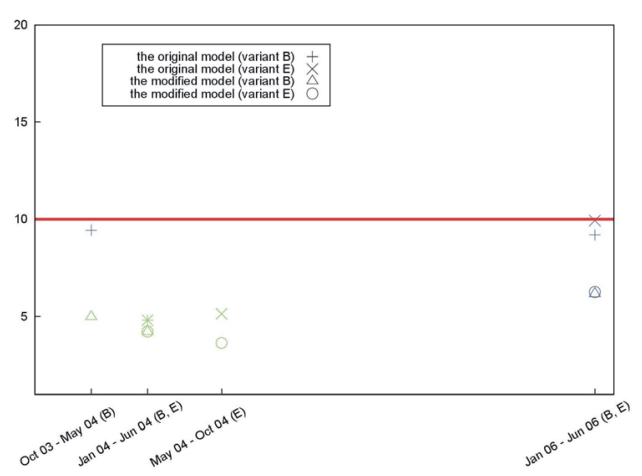

Fig. 10: The mean absolute percentage errors within price increase.

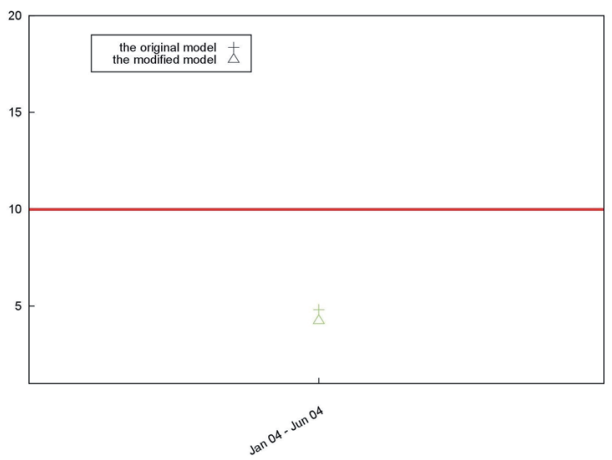

Fig. 11: The mean absolute percentage errors within trouble free forecasting terms - variant $B$.

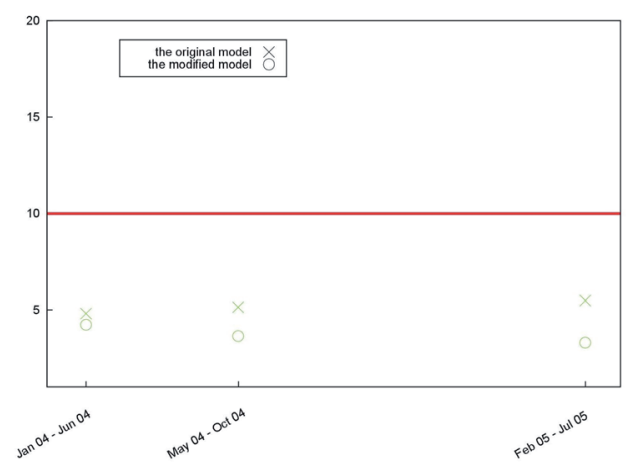

Fig. 12: The mean absolute percentage errors within trouble free forecasting terms - variant $E$.

(1 term) or forecasting terms with a small error (2 terms in which significant price fluctuation appeared) were obtained (Figure 10).

Within the forecasting terms with a small error, changes in price evolution also appeared. It could be seen a rapid decrease of the stock exchanges, even though at their beginning there was aluminium price increase. The forecasting was problematic considering previous price increase within the 
approximation term. Thereby the prognoses were increasing while the stock exchanges were steeply decreasing, so the errors in prognoses became higher and the initial condition drift occurred. By using the most successful drift (drift to the limiting month using the limiting value error $7 \%$ ) the initial condition value was the nearest to the prices in decline. Thus, the critical values were eliminated (2 terms) or reduced from two to one (1 term) as can be seen in Figure 8. In case of price increase after price decline, slower increasing prognoses obtained lower values than quicker increasing stock exchanges. This is why the forecasting accuracy was decreasing with time. If within the forecasting terms with a small error a moderate price increase after its decline occurred, the most successful initial condition drift (drift to the limiting month using the limiting value error $7 \%$ ) made the forecasting more so accurate that no critical value was acquired and trouble free forecasting terms were gained (2 terms). In the forecasting terms with steeper increase of stock exchanges after price decline, one critical value remained after the most successful initial condition drift. Thus, the forecasting terms remained in the group of forecasting terms with a small error (2 terms), see in Figure 9.

By having analyzed the aluminium price evolution we found out that the trouble free forecasting terms were typical of moderate price increase with its occasional oscillation. Bigger prognoses errors were caused by the change of the increase rate of the forecast stock exchanges in comparison to the increase rate of the stock exchanges within the approximation term. Using the initial condition drift the prognoses got closer to increasing stock exchanges, so the forecasting became more accurate. The mean absolute percentage error of forecasting term always reduced (see Figure 8, Figure 10, Figure 11 and Figure 12).

\section{Conclusions}

Forecasting movements of prices on the commodity exchanges was started from the original model calculating the prognoses within six months following the approximation term after the modification of the initial condition value by obtained monthly price prognoses. Even though the original model forecasted the stock exchanges reliably within the stable price course, within the rapid increase or decrease of stock exchanges, but also in the case of changes in the price course the forecasting failed. Since the variability with rapid and sudden changes is typical of the commodity price course, it's necessary search the possibility of making the forecasting more accurate. By chosen strategy of replacing the initial condition value by appropriate stock exchange the most problematic forecasting terms with a big error were eliminated. Forecasting became more accurate in all forecasting terms, where the initial condition drift occurred. Using determined modification, MAPE and also number of critical value were reduced. With regard to the chosen results, the strategy of initial condition drift significantly contributes to prognoses accuracy and it is a suitable way for improving original forecasting.

\section{Acknowledgments}

The research for this article was supported by Slovak VEGA Grant 1/0233/18.

\section{References and Notes}

[1] Geman, H., Smith, W.O. (2013). Theory of storage, inventory and volatility in the LME base metals. Resources Policy, Vol. 38, No. 1, p. 18-28.

[2] Heaney, R. (2002). Does knowledge of the cost of carry model improve commodity futures price forecasting ability? A case study using the London Metal Exchange lead contract. International Journal of Forecasting, Vol. 18, No. 1 , p. $45-65$.

[3] Rahamneh, Z., Reyalat, M., Sheta, A., Aljahdali, S. (2010). Forecasting stock exchanges using soft computing techniques. International Conference on Computer Systems and Applications, AICCSA, Hammamet.

[4] Bessec, M., Bouabdallah, O. (2005). What causes the forecasting failure of markov-switching models? A Monte Carlo study. Studies in Nonlinear Dynamics and Econometrics, Vol. 9, No. 2, 24 p.

[5] Feng, H. (2011). Forecasting comparison between two nonlinear models: Fuzzy regression versus SETAR. Applied Economics Letters, Vol. 18, No. 1, p. 1623-1627.

[6] Ismail, Z., Yahya, A., Shabri, A. (2009). Forecasting gold prices using multiple linear regression method. American Journal of Applied Sciences, Vol. 6, No. 8, p. 1509-1514.

[7] Sánchez Lasheras, F., de Cos Juez, F.J., Suárez Sánchez, A., Krzemién, A., Riesgo Fernández, P. (2015). Forecasting the COMEX copper spot price by means of neural networks and ARIMA models. Resources Policy, Vol. 45, p. 37-43.

[8] Varga, M. (2008). Forecasting commodity prices with exponential smoothing. Ekonomie a Management, Vol. 11, No. 3 , 
p. 94-97.

[9] Lascsáková, M. (2012). The study of numerical models' properties with the aim of improving accuracy of forecasts of commodity prices. Manufacturing and Industrial Engineering, Vol. 11, No. 1, p. 34-37.

[10] Lascsáková, M., Nagy, P. (2014). The aluminium price forecasting by replacing the initial condition value by the different stock exchanges. Acta Metallurgica Slovaca, Vol. 20, No. 1, p. 115-124.

[11] Lascsáková, M. (2015). Improving accuracy of the numerical model forecasting commodity prices. Applied Mechanics and Materials, Vol. 708, p. 251-256.

[12] Lascsáková, M. (2017). The influence of increasing sensitivity of the numerical model on the accuracy of the commodity price forecasting. Interdisciplinarity in Theory and Practice, No. 13, p. 5-10.

[13] http://www.Ime.com/home.asp, 16.2.2013

[14] Penjak, V., Lascsáková, M. (2001). Solution of the Cauchy problem for the ordinary differential equation $y^{\prime}=f(x, y)$ by means of the exponential approximation. Studies of University in Žilina, Vol. 1, p. 163-166.

\section{Biographical notes}

Mgr. Marcela Lascsáková, PhD., received PhD degree in Applied Mathematics at University of Žilina in 2009. She currently works as a senior lecturer at Department of Applied Mathematics and Informatics, Faculty of Mechanical Engineering, Technical University of Košice. Her research interests include numerical modelling, graph theory and combinatorics. 